\title{
Ruptured abdominal aortic aneurysm in Marfan's syndrome
}

\author{
K. Lafferty, Lesley McLean, J. Salisbury and L.T. Cotton \\ Kings College Hospital, Denmark Hill, London SE5 9RS, UK.
}

\begin{abstract}
Summary: A rare case of ruptured abdominal aortic aneurysm in Marfan's syndrome is described. The patient was treated successfully with a straight aortic tube graft: Histology of the aneurysm wall showed changes typical of cystic medial necrosis.
\end{abstract}

\section{Introduction}

In 1985 McKusick $^{1}$ described Marfan's syndrome as an 'abiotrophy' of connective tissue stating that; . . . the involved tissues wear out prematurely under the usual stresses and strains'. Cardiovascular complications are the predominant cause of death in these patients, aortic dissection ( $38 \%$ ), fusiform ascending aortic aneurysm (35\%) and mitral regurgitation $(22 \%)$ being the commonest post-mortem findings. ${ }^{2}$

Fusiform dilatation of the infra-renal abdominal aorta however would appear to be extremely rare. In an extensive review in 1982, Roberts \& $\mathrm{Honig}^{2}$ quoted only eight English language reports appearing since 1951. We report a patient with Marfan's syndrome who was treated successfully for a leaking abdominal aortic aneurysm.

\section{Case report}

A 32 year old man presented with a 12-hour history of severe abdominal and lumbar pain which had started acutely whilst gardening. He was of normal intelligence and had been diagnosed as having Marfan's syndrome as a child but had always enjoyed good health and took no medication. There was a strong family history with his father and six uncles suffering the same complaint but family ties had been lost many years previously and the patient was unaware of their whereabouts or state of health.

Correspondence: K. Lafferty, M.S., F.R.C.S.

Accepted: 2 February 1987

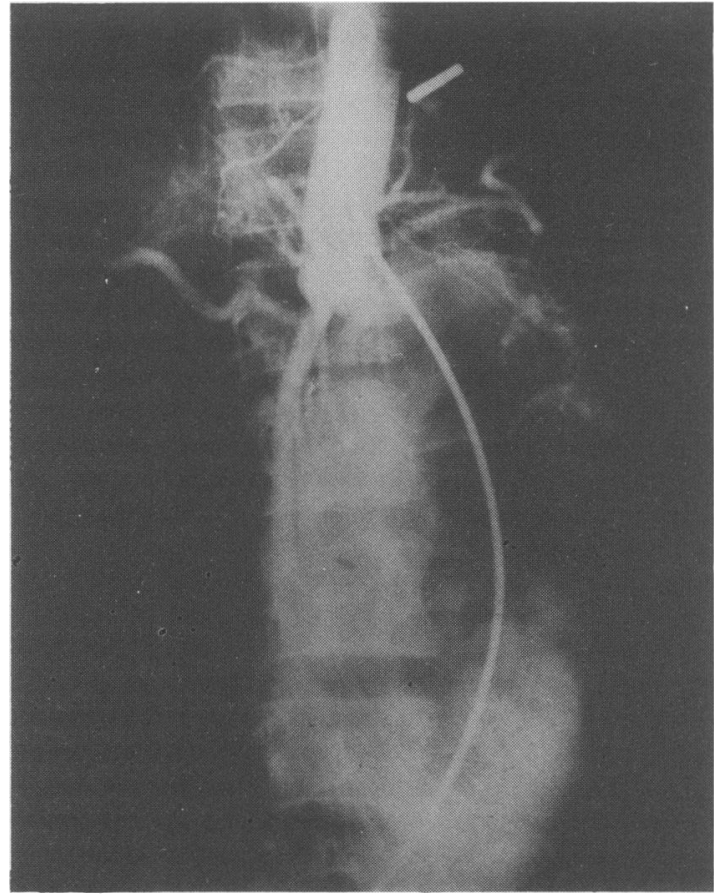

Figure 1 Trans-femoral aortogram.

On examination he was shocked, pale and oliguric with a large tender pulsatile mass extending from the left costal margin to the lower abdomen. All peri-

(C) The Fellowship of Postgraduate Medicine, 1987 


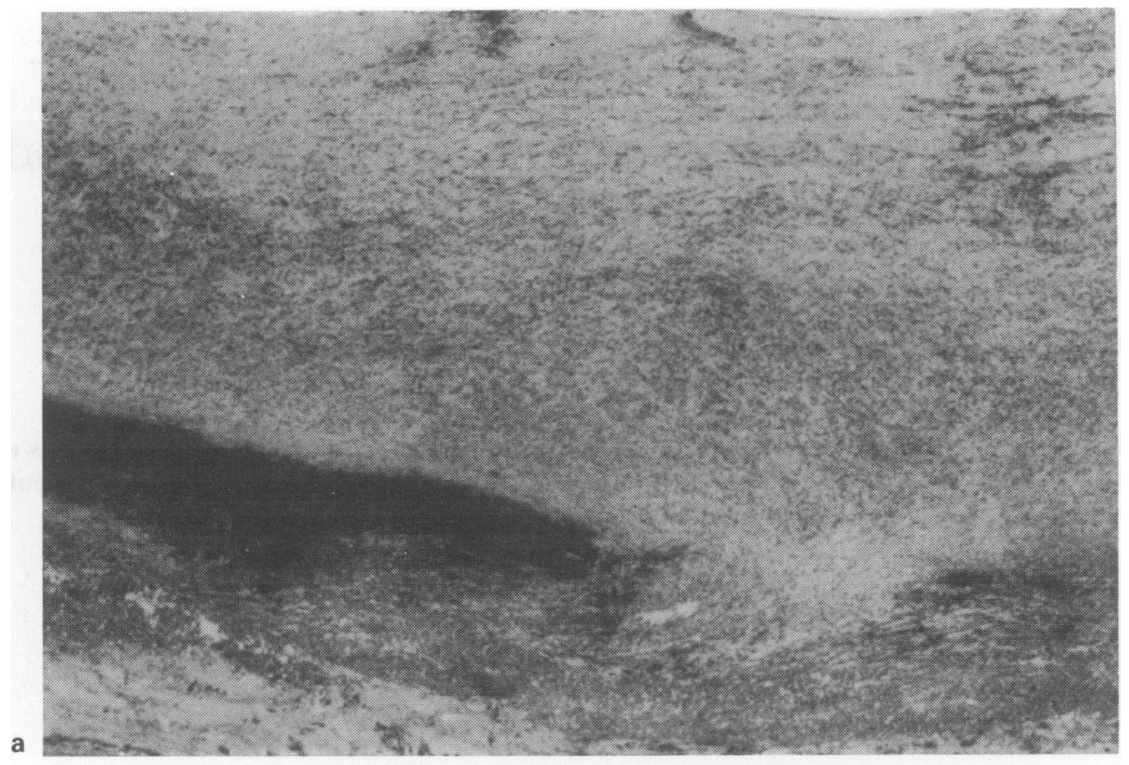

pheral pulses were present and equal. Examination of the chest revealed no murmurs or cardiomegaly. He was noted to be tall, slender and typically 'Marfanoid' with arachnodactyly, bilateral lenticular subluxation, myopia and a pigeon chest.

Ultrasound scan confirmed the presence of an aneurysm $11 \mathrm{~cm}$ in diameter associated with a large posterior haematoma. The aneurysm arose below the superior mesenteric artery with no extension into the chest but the renal arteries were not visualized Angiography (right transfemoral) showed that the aneurysm arose below the level of the renal arteries and that proximally the aorta was of normal calibres? (Figure 1). The condition of the patient precluded 
prolonging the procedure in order to obtain views of the ascending and thoracic aorta but a plain chest Xray showed no mediastinal widening.

At laparotomy the aneurysm extended from $1 \mathrm{~cm}$ below the left renal vein down to the aortic bifurcation sparing the iliac arteries and associated with a large retroperitoneal haematoma. After clamping, the sac was opened and found to contain fresh blood with no intraluminal thrombus and no evidence of atheroma, calcification, or dissection. In the posterior wall of the aneurysm there was a $10 \mathrm{~cm}$ longitudinal split running obliquely from left to right. This defect was lined posteriorly by pre-vertebral fascia and inferolaterally by the inferior vena cava.

An $18 \mathrm{~mm}$ woven Dacron straight tube graft was inlaid and anastamosed superiorly and inferiorly with continuous prolene. The non-dilated portions of aorta accepted sutures readily with no tendency to cut out. The aneurysm sac was used to cover the graft and was interposed inferiorly between the graft and vena cava. A biopsy of the aneurysm wall was taken. The patient made an uneventful post-operative recovery.

A subsequent echocardiogram showed that the patient's ascending aorta was moderately dilated at $5.2 \mathrm{~cm}$. The aortic and mitral valves were competent though the latter was described as 'floppy'. compared to a normal aorta. The paucity of darkly stained elastic fibres in the aneurysm section is clear. This appearance, classically termed Erdheim's cystic medial necrosis ${ }^{4}$ is, however, not confined to Marfan's syndrome and is seen commonly in normal aortas where it is now regarded as part of the normal ageing process: ${ }^{5}$ a view which supports McKusick's concept of premature atrophy.

The predominant involvement of the aortic root and ascending aorta in degenerative dilatation or dissection in Marfan's syndrome is well recognized. It is generally believed that this is because the pre-innominate ascending aorta is under maximal haemodynamic stress and is likely to suffer most in a condition characterized by a defect in the elastic fibre of connective tissue. ${ }^{1,2}$ Why a minority of patients with Marfan's syndrome present primarily with an abdominal aneurysm is unclear. Proposed aetiological factors concerning abdominal aneurysm formation include genetic predisposition, increased elastase and collagenase activity, copper deficiency, atheromatous degeneration, vasa vasora deficiency, decreased elastic lamellae and reflected standing waves ${ }^{6}$. Although Marfan's syndrome confers a genetic tendency to aneurysmal dilatation of the proximal aorta involvement of the abdominal portion presumably reflects the activity of an additional factor.

It is unusual to perform abdominal aortography in the acute situation and indeed under normal circumstances would result in an unnecessary and hazardous delay. In the present case however it was felt justified in order to exclude a dissection or thoracic extension and allow a planned procedure on an identified lesion. The patient remains well but is likely to need surgery to his ascending aorta in the future.

3. Goyette, E.M. \& Palmer, P.W. Cardiovascular lesions in arachnodactyly. Circulation 1953, 7: 373-379.

4. Erdheim, J. Medionecrosis aortae idiopathica. Virchows Arch (A) 1929, 273: 454-479.

5. Editorial. The ageing aorta. Lancet 1985, ii: 1402-1403.

6. Bergan, J.J. A personal view of abdominal aortic aneurysms. Br J Surg 1984, 71: 297-301. 